\title{
STM Probing of local oscillations of the Fano-Kondo effect: a Doniach-Sunjic approach for the Kondo peak
}

\author{
A. C. Seridonio ${ }^{1,2}$, L.N. Oliveira ${ }^{2}$, and M. Yoshida ${ }^{3}$ \\ ${ }^{1}$ ICCMP - International Center for Condensed Matter Physics, Universidade de Brasília, 04513, Brasília, DF, Brazil \\ ${ }^{2}$ Departamento de Física e Informática, Instituto de Física de São Carlos, \\ Universidade de São Paulo, 369, São Carlos, SP, Brazil \\ ${ }^{3}$ Departamento de Física, Instituto Geociências e Ciências Exatas, \\ Universidade Estadual Paulista, Rio Claro, SP, Brazil
}

(Received 01/07/2008)

\begin{abstract}
The use of the scanning tunneling microscope (STM) for the investigation of Kondo adatoms on normal metallic surfaces reveals a Fano-Kondo behavior of the conductance as a function of the tip bias. In this work, the Doniach-Sunjic expression is used to describe the Kondo peak and we analyze the effect of a complex Fano phase, arising from an external magnetic field, on the conductance pattern. It is demonstrated that such phase generates local oscillations of the Fano-Kondo line shape and can lead to the suppression of anti-resonances.
\end{abstract}

Keywords: STM (Scanning Tunneling Microscope), magnetic impurities, Kondo effect

\section{INTRODUCTION}

The electron transport through normal metallic junctions at zero temperature is governed by a tunneling transmission probability $T$ through an effective potential barrier formed in the contact region. In a single mode approximation, the conductance is given by the Landauer-Buttiker formula $G_{j u n c}=$ $\frac{2 e^{2}}{h} T$.

Magnetic impurities (or Kondo adatoms) on metal sample surfaces cause deviations of the conductance from this value as it was observed by many experimental groups that use the STM (Scanning Tunneling Microscope) techniques [1-3].

These investigations reveal the spatial distribution of the charge density oscillations around the Kondo adatom reflected in the tunneling conductance curves behavior around the Fermi level in tip bias measurements.

Usually the STM experiments are performed in systems with single $\mathrm{Co}$ atoms on $\mathrm{Au}(111)[1,2], \mathrm{Cu}(100)$ or $\mathrm{Cu}(111)$ surfaces [3] or with cobalt carbonyl complexes $\mathrm{Co}(\mathrm{CO})_{n}$ [4] and the manganese phthalocyanine ( $\mathrm{MnPc})$ magnetic molecules adsorbed on the top of $\mathrm{Pb}$ islands [5].

The main feature of such systems is the Fano-Kondo shape [6] for the conductance measured as a function of the tip bias. The corresponding mechanism is similar to those behind the anomalous transport properties in dilute magnetic alloys [7] , i.e., the Kondo effect. The latter is understood as an antiferromagnetic screening of the localized impurity by the host metallic electrons, efficient below the characteristic Kondo temperature $T_{K}$.

In this work, we consider a situation when a magnetic moment localized at the adatom site is coupled to two electron reservoirs: the conduction bands of the STM tip and of the host metal (Fig.(1)). The presence of these two couplings cause the Fano effect, initially observed in atomic physics [8]. It occurs due to the quantum interference between the electron tunneling from the adatom discrete level into the continuum of the STM tip and the host metal conduction bands. The interplay between these two effects is called Fano-Kondo effect.

We derive a formula for the tunneling conductance of the impurity-metal STM system in the Kondo regime. It is shown that the conductance strongly depends on the applied tip-host

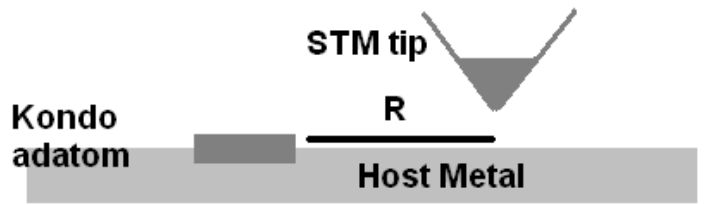

FIG. 1: Scanning Tunneling Microscope (STM) apparatus: a metallic tip above a host metal surface and lateral placed to a Kondo adatom. This system exhibits the Fano-Kondo effect.

metal bias, the tip-adatom lateral surrounding distance and the Fano factor for the interfering tunneling channels.

Based on studies of conductance through Aharonov-Bohm interferometers with embedded Quantum Dots [9, 10], we consider an imaginary Fano factor, arising from a magnetic field present in the tunneling gap region. The possibility of the appearance of an imaginary part in the Fano Factor for the STM system, was already pointed out in [2], but without deeper investigations.

In the present work, we describe the Kondo peak using Doniach-Sunjic expression, known from a previous Numerical Renormalization Group (NRG) analysis for the impurity spectral density $[11,12]$. It should be noted that alternative theoretical approaches, can be found in the references $[13,14]$.

We reveal a mechanism of local oscillations of the FanoKondo line shape, due to this complex Fano factor and establish the condition of the suppression of the Fano antiresonances for a certain value of the phase of the Fano factor.

\section{THE MODEL}

The description of the metallic host and its interaction with the adatom can be performed within the framework of the single impurity Anderson model [15] with a half-filled noninteracting conduction band. The conduction energies are measured from the Fermi level $(\varepsilon=0)$ and extend from $-D$ to $D$. The model Hamiltonian is 


$$
\begin{aligned}
H_{A} & =\sum_{\sigma} \int \varepsilon c_{\varepsilon \sigma}^{\dagger} c_{\varepsilon \sigma} d \varepsilon+\varepsilon_{d} \sum_{\sigma} d_{\sigma}^{\dagger} d_{\sigma}+U n_{d \uparrow} n_{d \downarrow} \\
& +\sqrt{\frac{\Gamma}{\pi}} \sum_{\sigma} \int d \varepsilon\left(c_{\varepsilon \sigma}^{\dagger} d_{\sigma}+H . c .\right) .
\end{aligned}
$$

The operator $c_{\varepsilon \sigma}$ of the conduction band is

$$
c_{\varepsilon \sigma}=\sum_{\vec{k}} c_{\vec{k} \sigma} \delta\left(\varepsilon-\varepsilon_{k}\right) / \sqrt{\rho_{0}} .
$$

with a host metal spin and energy-independent (flat band) density of states $\rho_{0}$ per spin. This is a reasonable approximation for the bias and temperature ranges considered, i.e., $|e V|<<D$ and $T<<T_{K}$ for a tip bias $V$.

The first term of (1) represents the surface free conduction states of the host metal band, the second and the third are the adatom Hamiltonian characterized by the electron occupation number $n_{d \sigma}=d_{\sigma}^{\dagger} d_{\sigma}$ for the spin $\sigma(\sigma=\uparrow, \downarrow)$ with energy $\varepsilon_{d}$ and Coulomb repulsion $U$.

The last term, proportional to $\Gamma$, corresponds to the local coupling between the adatom and the host metal and is responsible for a broadening of the discrete levels $\varepsilon_{d}$ and $\varepsilon_{d}+U$. In the Kondo regime, there is a third peak at the Fermi level, with a half-width given by $\Gamma_{K}=k_{B} T_{K}$.

The Anderson model (1) allows three possible configurations for charge fluctuations at the adatom site, the adatom can be empty, singly occupied with energy $\varepsilon_{d}$, or doubly occupied with energy $2 \varepsilon_{d}+U$.

The hopping between the conduction electrons and the valence states is characterized by the matrix element $v$, which allows charge transfer at the rate $\Gamma / \hbar$, with $\Gamma=\pi v^{2} \rho_{0}$.

In this paper, we focus on the conditions $\Gamma<<-\varepsilon_{d}, \varepsilon_{d}+$ $U$, and $\varepsilon_{d}<<\varepsilon_{F}, \varepsilon_{d}+U>>\varepsilon_{F}$, which exclude the empty and double adatom electronic occupation contributions to the system ground state.

For this range of the parameters, the Anderson model (1) can be mapped into the Kondo model by the use of the Schrieffer-Wolf transformation [16].

The full STM Hamiltonian $H_{S T M}$ consists of $H_{A}$ added to the tip conduction band $H_{t i p}$ and to the tunneling $H_{t u n}(\vec{R})$ Hamiltonians,

$$
H_{S T M}=H_{A}+H_{t i p}+H_{t u n}(\vec{R}),
$$

where $H_{t i p}$ is analogous to the first term on the right-hand side of the expression (1), i.e.,

$$
H_{t i p}=\sum_{\vec{p} \sigma} \varepsilon_{\vec{p}} a_{\vec{p} \sigma}^{\dagger} a_{\vec{p} \sigma}
$$

and $H_{t u n}(\vec{R})$ is

$$
H_{t u n}(\vec{R})=\sum_{\vec{p} \sigma}\left[t_{c} a_{\vec{p} \sigma}^{\dagger} B_{\sigma}(\vec{R})+H . c .\right] .
$$

The operators $a_{\vec{p} \sigma}$ describe the STM tip conduction states. The $B_{\sigma}(\vec{R})$ operator is

$$
B_{\sigma}(\vec{R})=\left[\int \tilde{N}_{\varepsilon}^{-1} \tilde{C}_{\varepsilon \sigma} d \varepsilon+q_{R} \sqrt{\pi \Gamma \rho_{0}} d_{\sigma}\right] / N_{q_{R}},
$$

with $\tilde{C}_{\varepsilon}$ given by

$$
\tilde{C}_{\varepsilon \sigma}=\tilde{N}_{\varepsilon} \sum_{\vec{k}} \varphi_{\vec{k}}(\vec{R}) c_{\vec{k} \sigma} \delta\left(\varepsilon-\varepsilon_{k}\right)
$$

The normalization factors are

$$
\begin{gathered}
\tilde{N}_{\varepsilon}=\left[\sum_{\vec{k} \sigma}\left|\varphi_{\vec{k}}(\vec{R})\right|^{2} \delta\left(\varepsilon-\varepsilon_{k}\right)\right]^{-1 / 2}, \\
N_{q_{R}}=\sqrt{1+\left|q_{R}\right|^{2}}
\end{gathered}
$$
$\vec{R}$

Here, $\varphi_{\vec{k}}(\vec{R})$ is a host conduction wavefunction at a position

Following the procedure of the works $[9,10]$, we account for a magnetic flux by adding complexes phases in the hopping terms of the Hamiltonian (5). Therefore, we define according to Ref. [17],

$$
q_{R}=\left(\pi \Gamma \rho_{0}\right)^{-1 / 2} \frac{t_{d R}}{t_{c}}=\left|q_{R}\right| \exp (i \phi),
$$

as the position dependent complex Fano factor, given by the ratio between the tip-adatom coupling $t_{d R}$ and the tip-host metal coupling $t_{c}$.

The Fano parameter, as we shall see, monitors the quantum interference between the tunneling channels [18].

The hopping term $t_{d R}$ vanishes at $R \rightarrow \infty$ and ensures the boundary STM adatom free tunneling condition $G_{j u n c}=$ $\frac{2 e^{2}}{h} T$, with $T=2\left|\pi t_{c} \rho_{t i p}\right|^{2}$ as the transmission probability of the STM tip-host metal potential barrier.

\section{THE METHOD}

We treat the STM having a tip-host metal weak energyindependent coupling $\left|t_{c}\right|$. The conductance formula is calculated up to the second order in $\left|t_{c}\right|$, with $H_{t u n}(\vec{R})$ being considered perturbatively [17]. Therefore, the tunneling conductance is given by

$$
G=G_{\max } \sum_{\sigma} \int \rho_{D O S \sigma}\left(\varepsilon+e V, T, R, q_{R}\right)\left(-\frac{\partial}{\partial \varepsilon} f(\varepsilon)\right) d \varepsilon
$$

where we defined $G_{\max } \equiv N_{q_{R}}^{2} \rho_{\text {tip }}^{-1} G_{j u n c}$ and

$$
\rho_{D O S \sigma}\left(\varepsilon, T, R, q_{R}\right)=-\frac{1}{\pi} \mathfrak{I}<<B_{\sigma}(\vec{R}) \mid B_{\sigma}^{\dagger}(\vec{R})>>_{\varepsilon},
$$

as the position dependent spectral density of states, determined from the imaginary part $\mathfrak{I}$ of the retarded Green's 
function $<<B_{\sigma}(\vec{R}) \mid B_{\sigma}^{\dagger}(\vec{R})>_{\varepsilon}$ thermally averaged over the eigenstates of the Hamiltonian (1).

The density of states (per spin) $\rho_{t i p}$ is also taken as a flat band with a value equal to the host metal conduction band density of states (an extended procedure for the derivation of the conductance formula can be found in the work done by Wein Fan [19]).

Looking to the expression (7), one sees that the tunneling conductance depends on non-orthonormal fermionic operators (6), i.e.,

$$
\left\{\tilde{C}_{\varepsilon \sigma}^{\dagger}, c_{\varepsilon^{\prime} \sigma}\right\}=\frac{\tilde{N}_{\varepsilon}}{\sqrt{\rho_{0}}} \delta\left(\varepsilon-\varepsilon^{\prime}\right) F_{\vec{R}}(\varepsilon)
$$

with a spatial function $F_{\vec{R}}(\varepsilon)$ given by

$$
F_{\vec{R}}(\varepsilon)=\sum_{\vec{k}} \varphi_{\vec{k}}(\vec{R}) \delta\left(\varepsilon-\varepsilon_{k}\right)
$$

The operator (6) can be expressed in terms of fermionic operators orthonormal to the $c_{\varepsilon \sigma}$.

Such operators will split the conductance into a background contribution dependent on the tip-adatom lateral distance, direct tunnelings from the tip-host metal couplings and their quantum interference.

We perform this procedure using the operator

$$
\tilde{c}_{\varepsilon \sigma}=N_{0}\left(\tilde{C}_{\varepsilon \sigma}-\frac{\tilde{N}_{0}}{\sqrt{\rho_{0}}} F_{\vec{R}}(0) c_{\varepsilon \sigma}\right),
$$

with a normalization factor evaluated at the Fermi level $\varepsilon=0$, i.e.,

$$
N_{0}=\left\{1-\left|\frac{\tilde{N}_{0}}{\sqrt{\rho_{0}}} F_{\vec{R}}(0)\right|^{2}\right\}^{-1 / 2}
$$

This leads to the following expression for $B_{\sigma}(\vec{R})$

$$
\begin{aligned}
& B_{\sigma}(\vec{R}) \\
& \quad=\left[\frac{\int d \varepsilon \tilde{c}_{\varepsilon \sigma}}{\tilde{N}_{0} N_{0}}+\sqrt{2} \frac{F_{\vec{R}}(0)}{\rho_{0}} f_{0 \sigma}+q_{R} \sqrt{\pi \Gamma \rho_{0}} d_{\sigma}\right] / N_{q_{R}},
\end{aligned}
$$

where we introduced the normalized fermionic operators $f_{0 \sigma}$ associated with a localized conduction state centered at the impurity site as

$$
f_{0 \sigma}=\sqrt{\frac{\rho_{0}}{2}} \int c_{\varepsilon \sigma} d \varepsilon .
$$

Details of the procedure can be found in Ref.[20].

As the Kondo effect occurs at low temperatures $\left(T_{K}>>\right.$ $T \rightarrow 0$ ), we consider small thermal energies neglecting temperature effects due to the Fermi surface broadening, replacing the Fermi Dirac derivative by the delta function and substituting $\rho_{D O S \sigma}\left(\varepsilon, T, R, q_{R}\right)$ by $\rho_{D O S \sigma}\left(\varepsilon, T=0, R, q_{R}\right)$ in the integral on the right-side of (11).
Defining $\tan \delta_{q} \equiv\left|q_{R}\right|$, we finally find a tunneling conductance expression at the tip bias

$$
G\left(e V, T<<T_{K}, R, q_{R}\right)=G_{\max } \sum_{\sigma} \rho_{D O S \sigma}
$$

where

$$
\begin{aligned}
\rho_{D O S \sigma} / \rho_{0} & =\left[1-\left(F_{\vec{R}}(0) / \rho_{0}\right)\right] \cos ^{2} \delta_{q_{R}}^{2}+\sin ^{2} \delta_{q} \sin ^{2} \delta_{\varepsilon} \\
& +2\left[F_{\vec{R}}(0) / \rho_{0}\right] \cos \phi \\
& \times \sin \delta_{q} \cos \delta_{q} \sin \delta_{\varepsilon} \cos \delta_{\varepsilon} \\
& +\left[F_{\vec{R}}(0) / \rho_{0}\right]^{2} \cos ^{2} \delta_{q} \cos ^{2} \delta_{\varepsilon}
\end{aligned}
$$

In (20), we used zero temperature Anderson Model Green's function identities [7, 15] and Doniach-Sunjic spectral density [11]

$$
\rho_{d \sigma}(\varepsilon)=\frac{1}{\pi \Gamma} \Re\left[\frac{i \Gamma_{K}}{\varepsilon+i \Gamma_{K}}\right]^{\frac{1}{2}}=\frac{1}{\pi \Gamma} \sin ^{2} \delta_{\varepsilon}
$$

for description of the Kondo peak

The terms proportional to $\sin ^{2} \delta_{\varepsilon}$ and $\cos ^{2} \delta_{\varepsilon}$ represent direct tunnelings from the STM tip to the adatom and to the host metal conduction band, respectively.

The interference between the tunneling channels is given by the mixture term $\sin \delta_{\varepsilon} \cos \delta_{\varepsilon}$.

The Doniach-Sunjic expression (21) for the Kondo peak was used before for the determination of the specific heat, the magnetic susceptibility and the electrical resistivity of the Single Impurity Anderson Model [12]. And more recently, it was employed to fit the experimental data for the tunneling conductance of individual manganese phthalocyanine (MnPc) molecules adsorbed on the top of $\mathrm{Pb}$ islands [5].

The lateral tip-distance conductance depends on the choice of the set of the wavefunctions $\varphi_{\vec{k}}(\vec{R})$, specific for each system of interest. However, at $R=0$ the behavior is spatial independent. Therefore, we derive a tunneling conductance behavior dictated by the following spectral density

$$
\begin{aligned}
\rho_{D O S \sigma} / \rho_{0} & =\cos ^{2} \frac{\phi}{2} \cos ^{2}\left(\delta_{e V}-\delta_{q}\right) \\
& +\sin ^{2} \frac{\phi}{2} \cos ^{2}\left(\delta_{e V}+\delta_{q}\right),
\end{aligned}
$$

where we used from (14), $F_{R=0}(0)=\rho_{0}$.

The expressions (19) and (22) are the main result of this work.

\section{ANALYSIS}

Real Fano Parameter-If $\phi=0$, the conductance depends only on the phase shift $\delta_{e V}-\delta_{q}$ as can be seen from (22). The left side of Fig. 2 shows the conductance as a function of the tip bias. The case $q=0.001$ corresponds to the weakcoupling situation $(q \rightarrow 0)$ between the adatom and the STM tip, where the tunneling into the host metal is suppressed by the screening around the adatom localized magnetic moment. 


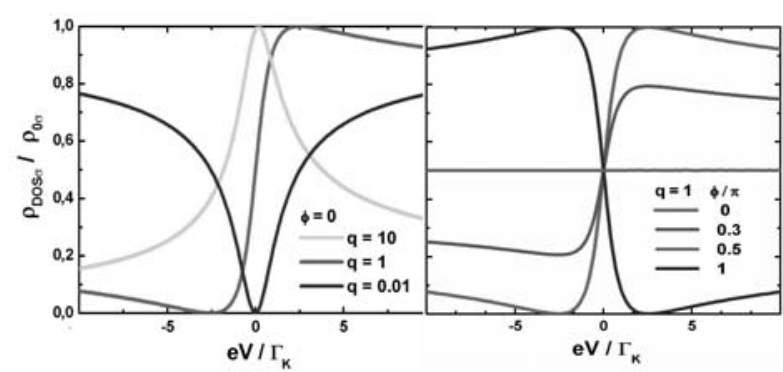

FIG. 2: Results at $T_{K}>>T \rightarrow 0$ using the Doniach-Sunjic description for the Kondo peak. The left plot shows different real Fano parameters for the spectral density $\rho_{D O S \sigma} / \rho_{0 \sigma}$ at $R=0$ against $e V / \Gamma_{K}$, where $\rho_{0 \sigma}=\rho_{0}$. The Fano-Kondo line shape appears for $q=1$. The right plot shows different complex Fano parameters for the spectral density $\rho_{D O S \sigma} / \rho_{0 \sigma}$ at $R=0$ against $e V / \Gamma_{K}$. For $q=1$ and for a given voltage, we note that the Fano-Kondo line shape oscillates due to the variation of the phase $\phi$ with a suppression given by the condition $\phi=\pi / 2$.

In the case $q=10$ (strong-coupling between the adatom and the STM tip, i.e., $q \rightarrow \infty$ ) the screening enhances the tunneling into the adatom and recovers the Kondo peak at the Fermi level.

The intermediate case $q=1.0$ corresponds to the two tunneling channels opened, which appears as a Fano-Kondo line shape. In this configuration, the tunneling competition from the tip into the host metal conduction band and into the adatom occurs.

Complex Fano Parameter-The right side of Fig. 2 shows the effect of the phase $\phi$ of the Fano factor on the conductance pattern. We see that with the increase of the Fano phase, the Fano-Kondo line becomes flattened until is transformed into a horizontal line tip bias independent, where the Fano-Kondo effect is thus suppressed for $\phi=\pi / 2$. In this regime, the interference between the tunneling channels are canceled and the conductance through a normal metallic junction is recovered, i.e., $G(e V, R=0, \phi=\pi / 2)=G_{j u n c}$. For $\phi>\pi / 2$, the competition between the tunneling channels is reversed and a symmetric behavior is observed.

We point out that the tune of the Fano phase factor turns the STM tunneling conductance similar to the conductance measured through a quantum dot in Aharonov-Bohm interferometers, as a function of the quantum dot level position $[9,10]$.

\section{v. CONCLUSIONS}

We have shown that a complex Fano factor, due to an external magnetic field, gives local oscillations for the STM tunneling conductance. For $\phi=\pi / 2$, the Fano-Kondo effect is totally suppressed and the conductance becomes tip bias independent (Fig.2).

\section{ACKNOWLEDGMENT}

We thank Drs. I.A. Shelykh and Valter Libero for a critical reading of the text. The work was supported by CNPq, FAPESP (01/14974-0; 04/08928-3) and IBEM Brazilian agencies.
[1] V. Madhavan, W. Chen, T. Jamneala, M. F. Crommie and N.S. Wingreen, Science 280, 567 (1998).

[2] V. Madhavan, W. Chen, T. Jamneala and F. Crommie, Phys. Rev.B 64, 165412 (2001).

[3] Nikolaus Knorr, M. Alexander Schneider, Lars Diekhöner, Peter Wahl and Klaus Kern, Phys. Rev. Lett. 88, 096804 (2002).

[4] P. Wahl, L. Diekhöner, G. Wittich, L. Vitali, M.A. Scheneider and K. Kern, Phys. Rev. Lett. 95, 166601 (2005).

[5] Ying-Shuang Fu, Shuai Hua Ji, Xi Chen, Xu-Cun Ma, Rui Wu, Chen-Chen Wang, Wen-Hui Dua, Xia-Hui Qiu, Bo Sun, Ping Zhang, Jin Feng Jia and Qi-Kun Xue, Phys. Rev. Lett. 99, 256601 (2007).

[6] O. Újághy, J. Kroha, L. Szunyogh and A. Zawadowski, Phys. Rev. Lett. 18, 2557 (2000).

[7] A.C. Hewson, The Kondo Problem to Heavy Fermions (Cambridge University Press, Cambridge, 1993).

[8] U. Fano, Phys. Rev. 124, 1866 (1961).

[9] Tae-Suk Kim and S.Hershfield, Phys. Rev. Lett. 88, 136601
(2002).

[10] W. Hofstetter, J. König and H. Shoeller, Phys. Rev. Lett. 87, 156803 (2001).

[11] H.O. Frota and L.N. Oliveira, Phys. Rev. B 33, 7871 (1986).

[12] H.O. Frota, Phys. Rev. B 45, 1096 (1992).

[13] J. Merino and O. Gunnarsson, Phys. Rev. B 69, 115404 (2004).

[14] H.G. Luo et al, T. Xiang, X.Q. Wang, Z.B. Su and L. Yu, Phys. Rev. Lett. 92, 256602 (2004).

[15] P.W. Anderson, Phys. Rev. 124, 41 (1961).

[16] J. R. Schrieffer and P.A. Wolf, Phys. Rev. 149, 491 (1966).

[17] A. Schiller and S. Hershfield, Phys. Rev. B 61, 9036 (2000).

[18] A.C. Seridonio, M. Yoshida and L.N. Oliveira, arXiv.org:condmat/0701529.

[19] Wei Fan, Phys. Rev. B 73, 045429 (2006).

[20] M. Yoshida, M. A. Whitaker and L.N. Oliveira, Phys. Rev B 41, 9403 (1990). 